\title{
Pulvinar projections to the striatum and amygdala in the tree shrew
}

\author{
1 Department of Anatomical Sciences and Neurobiology, University of Louisville Medical Center, Louisville, KY, USA \\ 2 Department of Anatomy, Chiang Mai University, Chiang Mai, Thailand \\ ${ }^{3}$ Department of Psychological and Brain Sciences, University of Louisville, Louisville, KY, USA
}

Jonathan D. Day-Brown', Haiyang Wei ${ }^{1}$, Ranida D. Chomsung1,2, Heywood M. Petry ${ }^{3}$ and Martha E. Bickford ${ }^{1 *}$

\section{Edited by:}

Jose L. Lanciego, University of

Navarra, Spain

Reviewed by:

Jose L. Lanciego, University of Navarra, Spain

Peter Redgrave, The University of Sheffield, UK

\section{${ }^{*}$ Correspondence:}

Martha E. Bickford, Department of Anatomical Sciences and

Neurobiology, School of Medicine,

University of Louisville, 500 S. Preston

St., Louisville, KY 40292, USA.

e-mail: martha.bickford@louisville.edu
Visually guided movement is possible in the absence of conscious visual perception, a phenomenon referred to as "blindsight." Similarly, fearful images can elicit emotional responses in the absence of their conscious perception. Both capabilities are thought to be mediated by pathways from the retina through the superior colliculus (SC) and pulvinar nucleus. To define potential pathways that underlie behavioral responses to unperceived visual stimuli, we examined the projections from the pulvinar nucleus to the striatum and amygdala in the tree shrew (Tupaia belangeri), a species considered to be a prototypical primate. The tree shrew brain has a large pulvinar nucleus that contains two SC-recipient subdivisions; the dorsal (Pd) and central (Pc) pulvinar both receive topographic ("specific") projections from SC, and Pd receives an additional non-topographic ("diffuse") projection from SC (Chomsung et al., 2008). Anterograde and retrograde tract tracing revealed that both $\mathrm{Pd}$ and $\mathrm{Pc}$ project to the caudate and putamen, and $\mathrm{Pd}$, but not $\mathrm{Pc}$, additionally projects to the lateral amygdala. Using immunocytochemical staining for substance $P(S P)$ and parvalbumin $(P V)$ to reveal the patch/matrix organization of tree shrew striatum, we found that SP-rich/PV-poor patches interlock with a PV-rich/SP-poor matrix. Confocal microscopy revealed that tracer-labeled pulvino-striatal terminals preferentially innervate the matrix. Electron microscopy revealed that the postsynaptic targets of tracer-labeled pulvino-striatal and pulvino-amygdala terminals are spines, demonstrating that the pulvinar nucleus projects to the spiny output cells of the striatum matrix and the lateral amygdala, potentially relaying: (1) topographic visual information from SC to striatum to aid in guiding precise movements, and (2) non-topographic visual information from SC to the amygdala alerting the animal to potentially dangerous visual images.

Keywords: blindsight, superior colliculus, striosome, matrix, synapse

\section{INTRODUCTION}

The primary visual pathway (from the retina to the lateral geniculate nucleus to the striate cortex or V1) is chiefly responsible for the conscious perception of visual stimuli. A second visual pathway relays presumably unconscious visual information from the retina to the superior colliculus (SC), then to the pulvinar nucleus. The pulvinar nucleus subsequently projects to visual areas of the parietal and temporal cortex (Stepniewska et al. 1999, 2000; Wong et al., 2009; Berman and Wurtz, 2010; Lyon et al. 2010) as well as to the striatum (Beckstead, 1984; Lin et al., 1984; Takada et al., 1985a,b; Harting et al., 2001a,b; Harting and Updyke, 2006; Kunzle, 2006) and to the amygdala (Linke et al., 1999; Shi and Davis, 2001). Although the exact functions of the pulvinar nucleus remain obscure, a variety of studies suggest that it plays important roles in directing spatial attention as well as linking visual stimuli with context-specific motor responses. Damage to, or inactivation of the pulvinar nucleus can result in inattention to the visual hemifield contralateral to the affected pulvinar, and/or impaired ability to guide movements in relation to visual signals (spatial neglect; Grieve et al. 2000; Arend et al., 2008; Snow et al., 2009; Wilke et al. 2010). Spatial neglect can also result from damage to either the cortical or subcortical targets of the pulvinar nucleus.
Lesion sites within the right parietal or temporal cortex can cause neglect symptoms (Verdon et al., 2010) and the subcortical damage sites most often associated with visual neglect are the right caudate and putamen (Karnath et al., 2002, 2004).

Visual pathways through the SC and pulvinar nucleus may also be responsible for (1) the ability of patients who have sustained V1 damage to continue to use visual cues to guide their movements even in the absence of conscious visual perception, a phenomenon referred to as "blindsight" (for review Cowey, 2010), and (2) the finding that fearful images can elicit emotional responses in the absence of their conscious perception, a phenomenon referred to as "unseen fear" or "subliminal fear" (Liddell et al., 2004; Williams et al., 2006). Findings supporting this function of the SC and pulvinar nucleus include evidence that lesions of the pulvinar impair responses to threatening visual images (Ward et al., 2005) and that the SC, pulvinar, and amygdala are activated during the brief presentation of fearful faces masked by neutral faces (Morris et al., 1999, 2001; Liddell et al., 2005).

The idea that components of the second visual pathway are involved in linking vision with action is also supported by correlating the anatomical organization of these pathways in different species with the behavioral effects of V1 lesions. In particular, in a 
prototypical primate, the tree shrew, the majority of the pulvinar nucleus receives input from the SC (Luppino et al., 1988; Chomsung et al., 2008), and visually guided movements are amazingly unrestricted even after complete removal of V1 (Diamond and Hall, 1969; Snyder et al., 1969). The tree shrew therefore provides an excellent model for the study of the organization of secondary visual pathways and their potential role in neglect, blindsight and/ or unseen fear.

In the tree shrew, the dorsal (Pd) and central (Pc) pulvinar both receive restricted, topographic ("specific") projections from the SC, and the $\mathrm{Pd}$ receives an additional widespread, non-topographic ("diffuse") SC projection (Luppino et al., 1988; Chomsung et al., 2008). Further distinctions between these pathways were provided by ultrastructural studies, which revealed a greater involvement of pulvinar interneurons in the specific pathway, when compared to the diffuse pathway (Chomsung et al., 2008). However, the function of these two pathways remains obscure; we found the $\mathrm{Pd}$ and $\mathrm{Pc}$ targeted similar regions of the temporal cortex (Chomsung et al., 2010).

In the current study, we sought to determine whether the $\mathrm{Pd}$ and Pc project differentially to the striatum and amygdala in order to gain insight into the potential function of the specific and diffuse tectopulvinar pathways. Within the striatum, we examined the relationship of $\mathrm{Pd}$ and $\mathrm{Pc}$ projections to the striosome and matrix compartments to further assess the function of these two pathways. Graybiel and Ragsdale (1978) originally described the unique organization of striatum sections stained for acetylcholinesterase (AChE) as a mosaic of pale staining patches (or striostomes) distributed in a more densely stained matrix. Subsequent studies of the connection patterns of these compartments suggest that they participate differentially in limbic-based (striosome) and sensorimotor/associative (matrix) circuits (for review, see Graybiel, 2008). Our results indicate that the specific tectopulvinar pathway relays topographic visual information to the striatum matrix while the diffuse tectopulvinar pathway relays non-topographic visual information to the amygdala. We suggest that the specific pathway may aid in the visual guidance of precise movements, while the diffuse pathway may function as an "alarm" pathway to potentially alert the animal to dangerous visual images.

\section{MATERIALS AND METHODS}

A total of 9 adult tree shrews (Tupaia belangeri); 4 male and 5 female, (140-270 g) were used for these experiments. To label pulvinar cells by retrograde transport, 2 tree shrews received an iontophoretic injection of the beta subunit of cholera toxin (CTB; List Biological Laboratories, Inc., catalog \#105) or fluorogold (FG; Fluorochrome LLC, Denver, CO, USA) in the putamen (1, CTB) or amygdala (1, CTB and FG on opposite sides). To label pulvinar projections by anterograde transport, 7 tree shrews received an iontophoretic injection of biotinylated dextran amine (BDA; 3,000 MW, Molecular Probes, Eugene, OR, USA) in the pulvinar nucleus (other pulvinar projections in these 7 cases were reported in our earlier publications (Chomsung et al., 2008, 2010). Selected sections from injected tree shrew brains were stained for acetylcholinesterase (AChE) to identify pulvinar subdivisions, or substance $\mathrm{P}(\mathrm{SP})$, parvalbumin, or the potassium channel interacting protein (KChIP) to identify the patch matrix compartments of the striatum.
All methods were approved by the University of Louisville Animal Care and Use Committee and conform to the National Institutes of Health guidelines.

\section{TRACER INJECTIONS}

Tree shrews that received injections were initially anesthetized with intramuscular injections of ketamine $(100 \mathrm{mg} / \mathrm{kg})$ and xylazine $(6.7 \mathrm{mg} / \mathrm{kg})$. Additional supplements of ketamine and xylazine were administered approximately every $45 \mathrm{~min}$ to maintain deep anesthesia through the completion of the injections. Prior to injection, the tree shrews were placed in a stereotaxic apparatus and prepared for sterile surgery. The heart rate was continuously monitored with a MouseOx pulse oximeter (STARR Life Sciences Corp., Pittsburgh, PA, USA). A small area of the skull overlying the putamen, amygdala or pulvinar nucleus was removed and the dura reflected. A glass pipette containing BDA ( $5 \%$ in saline, tip diameters approximately $3 \mu \mathrm{m}$ ) or FG ( $2 \%$ in saline, tip diameter approximately $10 \mu \mathrm{m})$ or CTB ( $1 \%$ desalted CTB in $0.1 \mathrm{M}$ phosphate buffer (PB), pH 6.0; tip diameters approximately $2 \mu \mathrm{m}$ ) was lowered vertically and the tracer was ejected iontophoretically $(2 \mu \mathrm{A}$ positive current for 15-30 $\mathrm{min}$ ).

After a 7-day survival period, the tree shrews were given an overdose of ketamine $(600 \mathrm{mg} / \mathrm{kg})$ and xylazine $(130 \mathrm{mg} / \mathrm{kg})$ and were perfused through the heart with Tyrode solution, followed by a fixative solution of $2 \%$ paraformaldehyde and $2 \%$ glutaraldehyde or $4 \%$ paraformaldehyde in $0.1 \mathrm{M} \mathrm{PB}$ ( $\mathrm{pH} 7.4$ ). The brain was removed from the skull, sectioned to a thickness of $50 \mu \mathrm{m}$ using a vibratome, and placed in $\mathrm{PB}$.

\section{HISTOCHEMISTRY TO REVEAL TRACERS}

The BDA was revealed by incubating sections in a 1:100 dilution of avidin and biotinylated horseradish peroxidase (ABC; Vector Laboratories, Burlingame, CA, USA) in phosphate-buffered saline (0.01 M PB with $0.9 \% \mathrm{NaCl}, \mathrm{pH} 7.4$; PBS) overnight at $4^{\circ} \mathrm{C}$. The sections were subsequently rinsed three times in $\mathrm{PB}$ (10 min each), reacted with nickel-intensified 3,3-diaminobenzidine (DAB) for $5 \mathrm{~min}$, and washed in PB. DAB-labeled sections were either mounted on slides or prepared for electron microscopy as described below. To reveal BDA for confocal microscopy (in combination with immunocytochemical labeling of the patch/matrix compartments, described below) sections were incubated in a 1:100 dilution of streptavidin conjugated to Alexa Fluor 488 or 546 (Molecular Probes, Eugene, OR, USA).

The FG was revealed with ultraviolet light, or by incubation with a rabbit anti-FG antibody (Fluorochrome) diluted 1:50,000. The antibody was revealed by either a biotinylated goat-anti-rabbit antibody (1:100, Vector), $\mathrm{ABC}$ and $\mathrm{DAB}$ reaction, or by a goat-anti-rabbit antibody conjugated to Alexa Fluor 488 or 546 (Molecular Probes). The CTB was revealed using a rabbit-anti-cholera toxin antibody (Sigma) diluted 1:10,000, followed by a biotinylated goat-anti-rabbit antibody (Vector), $\mathrm{ABC}$, and $\mathrm{DAB}$ reaction or a goat-anti-rabbit antibody conjugated to Alexa Fluor 488 or 546 (Molecular Probes).

A Neurolucida system and tracing software (MicroBrightField, Inc., Williston, VT, USA) was used to plot the distributions of pulvino-putamen and pulvino-amygdala cells and terminals. Adjacent sections stained for $\mathrm{AChE}$ (described below) were used to delineate the Pd and Pc. 


\section{AChE STAINING TO REVEAL PULVINAR SUBDIVISIONS}

Using a protocol modified from Stepniewska and Kaas (1997), the tissue was rinsed in deionized water, placed in a solution of AChE for $3 \mathrm{~h}$, and then rinsed in saline, followed by deionized water, before reacting with a $1.25 \%$ sodium sulfite solution for $1 \mathrm{~min}$. Following deionized water rinses, the tissue was then incubated in a $1 \%$ silver nitrate solution for $5 \mathrm{~min}$, rinsed with deionized water and placed in a $5 \%$ sodium thiosulfite solution to adjust the contrast of the tissue staining (approximately $5 \mathrm{~min}$ ). Finally, the tissue was rinsed in saline, and mounted on slides for light microscope examination.

\section{IMMUNOCYTOCHEMISTRY TO REVEAL PATCH/MATRIX PROTEINS}

To reveal the patch/matrix organization within the tree shrew striatum, antibodies shown to bind to proteins that are differentially expressed in the patch or matrix compartments of the striatum in various species were employed. The following antibodies and dilutions were used: rat-anti-substance P (SP; Accurate Chemical, Westbury, NY, USA used at a dilution of 1:250), mouse-anti-paravalbumin (Sigma, St Louis, MO, USA used at dilution of 1:10,000), mouse-anti-potassium channel interacting protein (KChIP; NeuroMab, UC Davis, used at a dilution of 1:5). Selected sections were incubated overnight in one of the above antibodies. The next day sections were rinsed in $0.1 \mathrm{M}$ phosphate buffer, incubated $1 \mathrm{~h}$ in the appropriate secondary antibody at a 1:100 dilution (biotinylated goat-anti-rat for substance P; biotinylated goat-anti-mouse for parvalbumin and KChIP; Vector Laboratories, Burlingame, CA, USA), rinsed again and incubated for $1 \mathrm{~h}$ in avidin and biotinylated-horseradish peroxidase (ABC solution) at a dilution of 1:100, before reacting with nickel-enhanced diaminobenzidine (DAB).

\section{CONFOCAL MICROSCOPY}

To determine if pulvino-striatal axons innervate the patch or matrix compartments or the striatum, sections containing terminals labeled by the anterograde transport of BDA injected in the pulvinar nucleus were incubated overnight in a 1:100 dilution of streptavidin conjugated to Alexa 546 (Molecular Probes, Carlsbad, CA, USA) and either the rat-anti-SP or mouse-anti-parvalbumin antibodies. The following day the sections were rinsed and incubated for $1 \mathrm{~h}$ in secondary antibodies (anti-rat for substance P; anti-mouse for parvalbumin) conjugated to Alexa Fluor 488 at a dilution of $1: 100$. Sections were then mounted and viewed with a laser scanning confocal microscope (Olympus 3 Laser Scanning Confocal Microscope Canter Valley, PA, USA). To illustrate the interlocking pattern of the patch and matrix compartments, sections were incubated in rat-anti-SP 1:250 and mouse-antiparvalbumin 1:10,000 overnight. The following day the sections were incubated in biotinylated goat-anti-rat for $1 \mathrm{~h}$, followed by streptavidin conjugated to Alexa Fluor 546 and anti-mouse conjugated to Alexa Fluor 488 for $1 \mathrm{~h}$. Sections were then mounted and viewed with the confocal microscope.

\section{ELECTRON MICROSCOPY}

Striatum and amygdala sections that contained terminals labeled by the anterograde transport of BDA were postfixed in $2 \%$ osmium tetroxide, dehydrated in an ethyl alcohol series, and flat embedded in Durcupan resin between two sheets of Aclar plastic (Ladd Research, Williston, VT, USA). Durcupan-embedded sections were first examined with a light microscope to select areas for electron microscopic analysis. Selected areas were mounted on blocks, ultrathin sections (70-80 nm, silver-gray interference color) were cut using a diamond knife, and sections were collected on Formvar-coated nickel slot grids. Selected sections were stained for the presence of gamma amino butyric acid (GABA). A postembedding immunocytochemical protocol described previously (Patel and Bickford, 1997) was employed. The GABA antibody (Sigma, catalog \# A2052, used at a dilution of 1:1000$1: 2000)$ was tagged with a goat-anti-rabbit antibody conjugated to 15-nm gold particles (Amersham, Arlington Heights, IL, USA). The sections were air dried and stained with a $10 \%$ solution of uranyl acetate in methanol for $30 \mathrm{~min}$ before examination with an electron microscope.

All labeled terminals involved in a synapse were photographed within each examined section. The pre- and postsynaptic profiles were characterized on the basis of size (measured using SigmaScan software, SPSS Inc., Chicago, IL, USA) and the presence or absence of synaptic vesicles.

\section{RESULTS \\ PD AND PC PROJECT TO THE STRIATUM; PD PROJECTS TO THE AMYGDALA}

Following large BDA injections that involved both the Pd and Pc (Figure 1A), terminals labeled by anterograde transport fill large regions of the postcommisural putamen (Figure 1B) caudate (Figure 1C), as well as the lateral amygdala (Figure 1F). No pulvino-striatal terminals were distributed in regions of the caudate and putamen rostral to the anterior commissure. Smaller injections, confined to either the Pd or Pc (example Pc injection illustrated in Figure 1D; all other injection sites illustrated in Chomsung et al., 2008, 2010), labeled more discrete clusters of terminals in the caudate (Figures $2 \mathrm{~A}-\mathrm{C}$ ) and putamen (Figures 1E, 2A-C, E-G), and injections of the Pd, but not the $\mathrm{Pc}$, also labeled diffusely distributed axons in the lateral amygdala (Figures 2E-G).

To confirm the differential projection patterns of the Pd and Pc, we placed injections of CTB or FG in the putamen or amygdala (Figure 2I). Confirming our anterograde tracing results, an injection in the putamen (Figure 2I, pink) that overlapped the distribution of terminals labeled by Pc BDA injections (Figures 2A-C) labeled clusters of cells in the Pc (Figure 2L) that were located in regions very similar to the location of the Pc BDA injections (Figure 2D). Similarly, injections in the amygdala (Figure 2I, green and orange) labeled cells in the Pd (Figures 2J,K) that were located in regions very similar to the location of the Pd BDA injections (Figure 2H).

\section{THE PATCH MATRIX ORGANIZATION OF THE TREE SHREW STRIATUM}

As illustrated in Figures 3 and 4, several different antibodies reveal the striosomes and matrix of the tree shrew caudate and putamen. An antibody against substance $\mathrm{P}$ stains dense patches of terminals (3A-C, G), while an antibody against parvalbumin intensely stained cells and neuropil within the matrix $(3 \mathrm{D}, \mathrm{E})$. Interdigitating substance $\mathrm{P}$ and parvalbumin staining patterns are illustrated in 


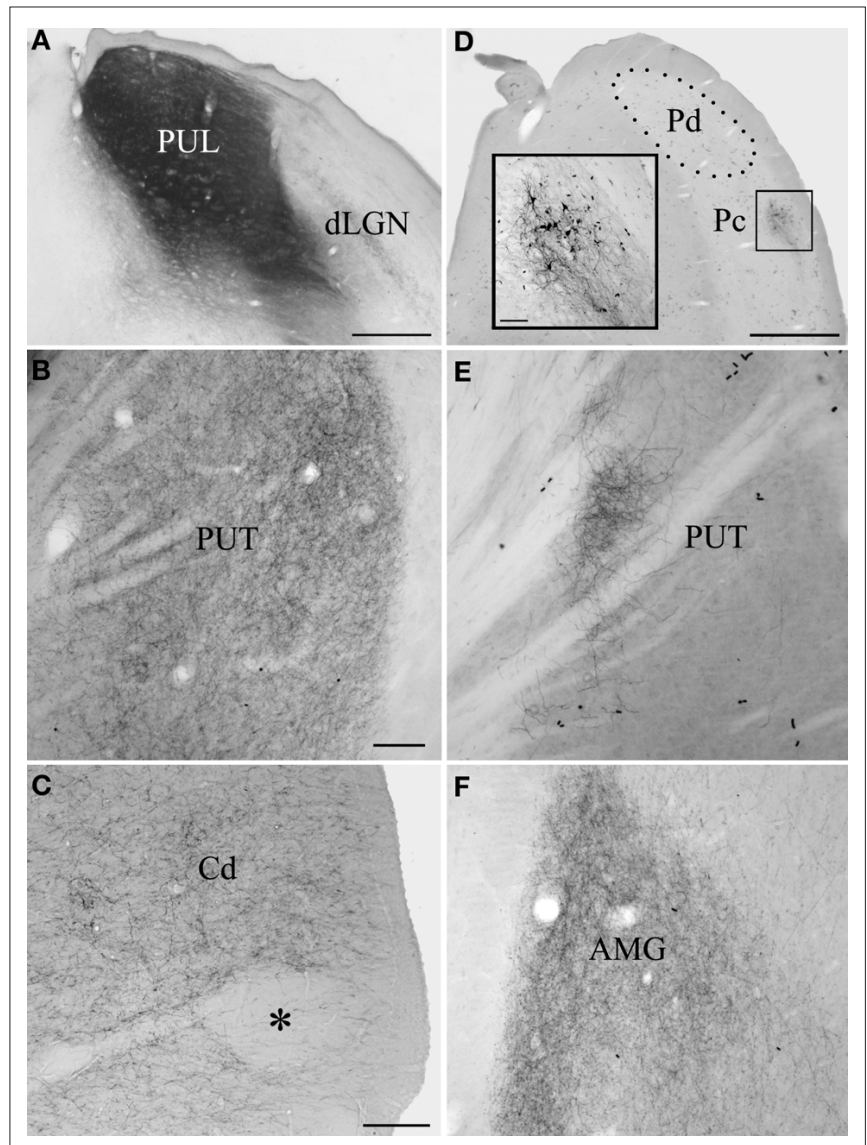

FIGURE 1 |The pulvinar nucleus projects to the striatum and lateral amygdala. Large BDA injections that involved both the dorsal $(\mathrm{Pd})$ and central (Pc) pulvinar (A) labeled terminals throughout putamen (PUT, B) and caudate nucleus $(\mathrm{Cd}, \mathbf{C})$, although conspicuous patches (asterisk) were devoid of label. The injection illustrated in (A) also labeled terminals throughout the lateral amygdala (AMG, F). Smaller BDA injections confined to the Pd or Pc labeled restricted clusters of terminals in the $\mathrm{Cd}$ and PUT. An example Pc injection is illustrated in (D) (inset illustrates the injection site at higher magnification) and the resulting terminal distribution in the PUT in (E). Scale bar in $\mathbf{A}=500 \mu \mathrm{m}$. Scale bar in $\mathbf{B}=100 \mu \mathrm{m}$ and also applies to (E). Scale bar in $\mathbf{C}=100 \mu \mathrm{m}$ and also applies to (F). Scale bar in $\mathbf{D}=1 \mathrm{~mm}$ (inset scale bar $=100 \mu \mathrm{m}$ ). dLGN, dorsal lateral geniculate nucleus; OT, optic tract. A was previously published in Chomsung et al., 2010.

Figures 4B-D. As recently demonstrated in primate tissue (Mikula et al., 2009), an antibody against KChIP stained cells throughout the matrix of the caudate and putamen and dense patches of terminals in striosomes $(3 \mathrm{~F})$ in the tree shrew striatum.

\section{THE PD AND PC PROJECT TO THE MATRIX OF THE STRIATUM}

The labeling patterns observed following the large injections of BDA in the pulvinar nucleus suggest that pulvino-striatal axons innervate the matrix. These injections labeled axons that innervated large regions of the caudate and putamen, but conspicuous patches were devoid of labeled axons (Figure 1C). To confirm whether these unlabeled zones corresponded to striosomes, we stained tissue containing BDA-labeled pulvino-striatal axons for substance $\mathrm{P}$ or parvalbumin. As illustrated in Figures 4E-J, all observed pulvinostriatal axons originating from either the Pd or Pc innervated the matrix, as defined by substance $\mathrm{P}$ or parvalbumin staining. We therefore conclude that the majority of pulvino-striatal axons innervate the matrix.

\section{THE PD AND PC CONTACT SPINES IN THE STRIATUM AND AMYGDALA}

Using electron microscopy, we examined a total of 181 pulvinoputamen terminals (labeled by the anterograde transport of BDA injected into the $\mathrm{Pd}$ and/or Pc) that were involved in synaptic contacts. No size differences were detected in the populations of terminals labeled by $\mathrm{Pd}, \mathrm{Pc}$, or combined $\mathrm{Pd} / \mathrm{Pc}$ injections (Pd injections: $0.48 \pm 0.30 \mu \mathrm{m}^{2}$, Pc injections: $0.47 \pm 0.24 \mu \mathrm{m}^{2}$, $\mathrm{Pd} / \mathrm{Pc}$ injections: $0.47 \pm 0.27 \mu \mathrm{m}^{2}$ ). Most terminals formed single synapses $(62 \%$, Figures 5A,B), but smaller numbers of perforated (34\%, Figure 5C) or multiple (4\%) synaptic contacts were also identified. Pulvino-putamen terminals primarily contacted spines (Pc, 100\%; Pd, 94\%; Pd/Pc, 93.3\%) as illustrated in Figures 5A-C. This suggests that pulvino-striatal terminals contact GABAergic spiny projection neurons. We attempted to confirm the GABAergic nature of the postsynaptic targets by using postembedding immunocytochemical techniques to reveal the presence of GABA. However, we did not observe any accumulation of GABA within spines, although GABAergic terminals were easily detected by a qualitative assessment of gold particle density (Figures 5A and G).

We examined a total of 123 pulvino-amygdala terminals (labeled by a combined $\mathrm{Pd} / \mathrm{Pc}$ injection) that were involved in synaptic contacts. The size of the labeled terminals $\left(0.42 \pm 0.17 \mu \mathrm{m}^{2}\right)$ was similar to the size of pulvino-putamen terminals. Most terminals formed single synapses (55\%, Figures 5D, F and G), but smaller numbers of perforated (35\%) or multiple (10\%; Figure $5 \mathrm{E})$ synaptic contacts were also identified. All pulvino-amygdala terminals were found to contact spines, as illustrated in Figures 5D-G.

\section{DISCUSSION}

Figure 6 schematically illustrates the potential influence of pulvinar projections on basal ganglia circuits and behavior initiated by the SC. The current results and those of our previous study of tectopulvinar projections (Chomsung et al., 2008) indicate that visual information from the SC is relayed via the pulvinar nucleus to the spiny output cells of the striatum matrix and the lateral amygdala. As discussed below, we suggest that the specific projections from the SC to the Pd and Pc relay topographic visual information to the striatum to aid in guiding precise movements related to pursuit of non-threatening objects, while the diffuse projections from the SC to the Pd relay non-topographic visual information to the amygdala to alert the animal to potential danger and initiate escape behavior.

\section{POTENTIAL RELATION OF PD AND PC PROJECTIONS TO APPROACH AND AVOIDANCE BEHAVIOR}

Stimulation studies in rats revealed that the SC initiates at least two distinct behaviors: stimulation of the lateral SC (representing the lower visual field) initiates orienting/approach behavior, and stimulation of the medial SC (representing the upper visual field) initiates escape/avoidance behavior (Sahibzada et al., 1986). Subsequent lesion studies revealed that these behaviors are mediated by two anatomically distinct cell groups (Redgrave et al., 


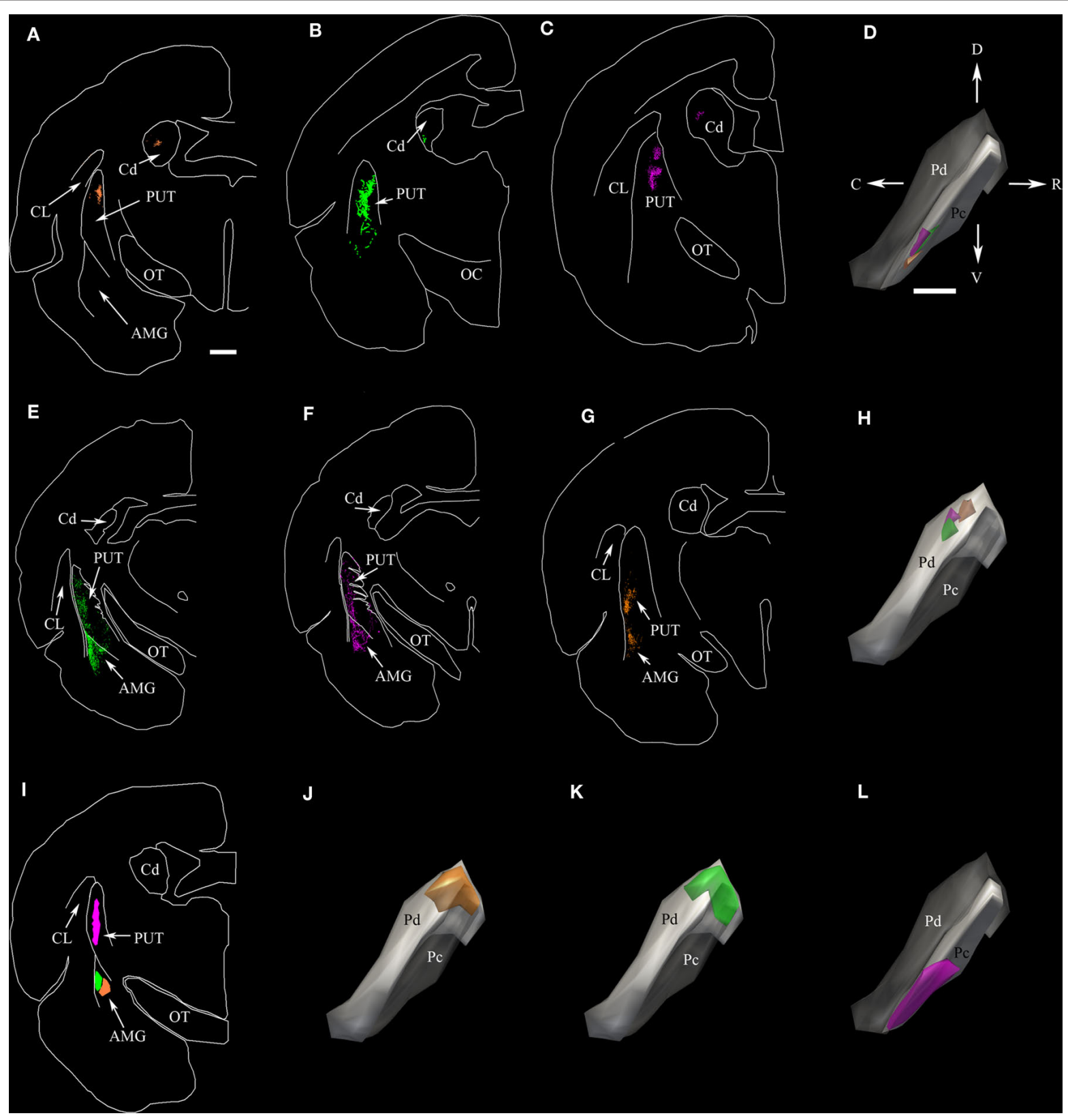

FIGURE 2 |The dorsal (Pd) and central (Pc) pulvinar project to the striatum; only the Pd projects to the amygdala. (A-C) Two dimensional (2D) neurolucida plots of the distribution of terminals labeled by anterograde transport in the caudate (Cd) and putamen (PUT) following three separate injections of BDA in the Pc. (D) Injection sites for (A-C) depicted in three-dimensional (3D) neurolucida reconstructions of the pulvinar nucleus. The color of each injection site corresponds to the color of the terminals in (A-C). (E-G) Two-dimensional plots of the distribution of terminals labeled by anterograde transport in the PUT and amygdala (AMG) following three separate injections of BDA in the Pd. (H) Three-dimension reconstructions of injection sites for (E-G). Because the full extent of the Pd is not viewable in the orientation depicted, the Pc subdivision was made transparent in this panel as well as in $(\mathbf{J}, \mathbf{K})$. (I) Two-dimension plots of the location of FG and CTB injections in the PUT and AMG. $(\mathbf{J}, \mathbf{K}, \mathbf{L})$

Three-dimension reconstructions of the distribution of cells labeled in the pulvinar nucleus by retrograde transport from the injections illustrated in I. The color of the cell distributions corresponds to the injection site colors in I. Scale bar in $\mathbf{A}=1 \mathrm{~mm}$ and applies to all 2D plots. Scale bar in $\mathbf{D}=1 \mathrm{~mm}$ and applies to all 3D pulvinar reconstructions. C, caudal, CL, claustrum, D, dorsal, OC, optic chiasm, OT, optic tract, R, rostral, $V$, ventral. Orientation arrows in D also apply to all 3D pulvinar reconstructions.
1986; Bickford and Hall, 1989) within the deep layers: approach movements by the crossed descending tecto-reticulo-spinal projection (predorsal bundle, $\mathrm{PDB}$ ) and avoidance behavior by the ipsilateral descending (cuneiform, CNF) pathway (Dean et al., 1986, 1989). The primary sensory responses of these two cell groups: $\mathrm{PDB}$ cells to somatosensory stimulation within the lower visual field, and CNF cells to large "looming" visual stimuli in the upper visual field, suggest that the PDB initiates 

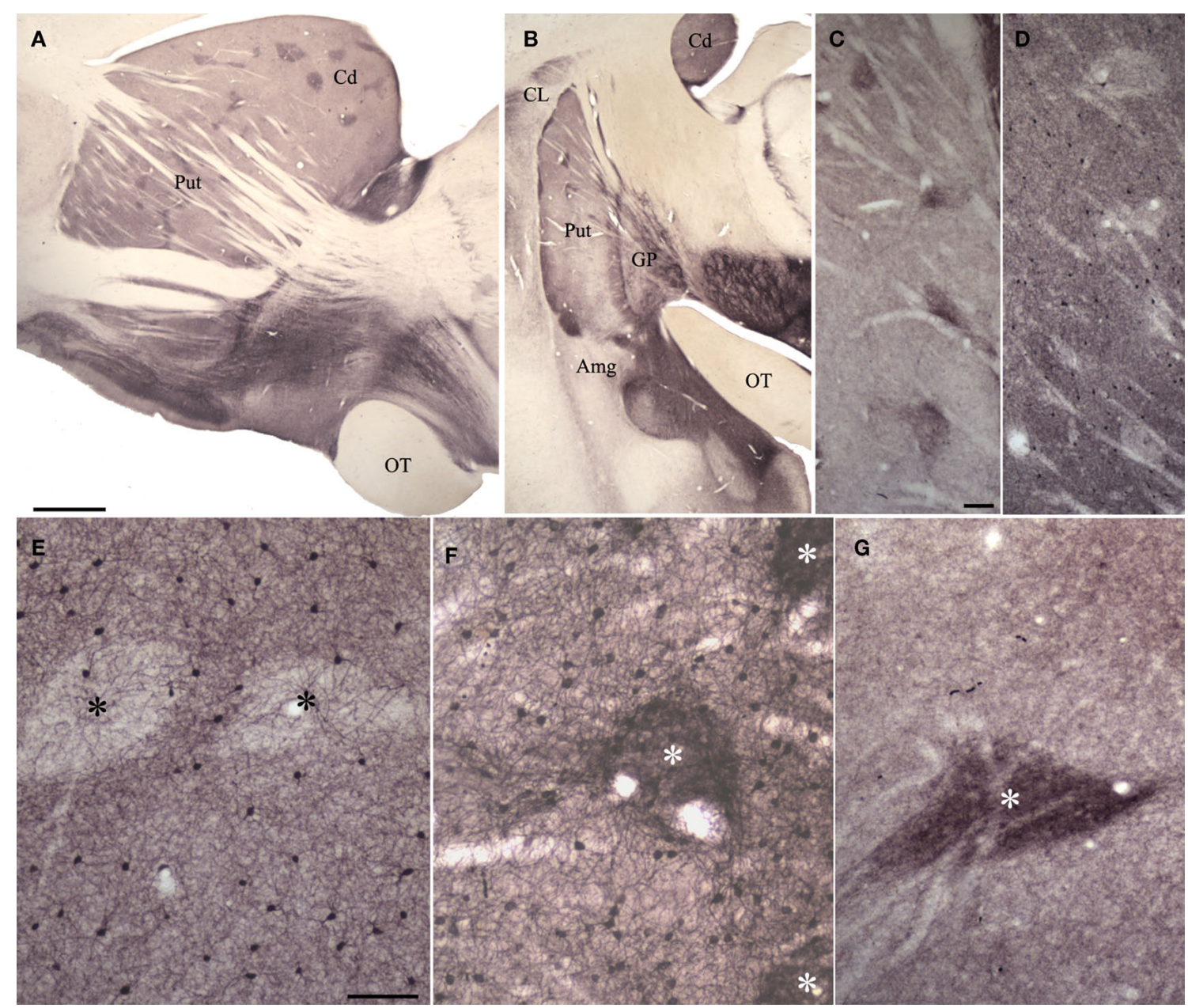

FIGURE 3 | Substance P (SP), parvalbumin (PV) and potassium channel interacting protein $(\mathrm{KChIP})$ antibodies reveal patch/matrix organization of tree shrew striatum. (A,B) Sections (parasagittal, $\mathbf{A}$; coronal, B) through the striatum stained for SP. Dark SP-positive patches are seen throughout the tree shrew striatum. (C,D) Adjacent sections through the putamen showing SP staining of patches (C) and PV staining in the matrix (D). (E) PV stains cells within the matrix but not within patches (black asterisks). (F) KChIP stains clusters of terminals in patches (white asterisk) and cells in the matrix. (G) SP stains densely distributed terminals in patches (white asterisk). In (A), scale bar $=1 \mathrm{~mm}$ and applies to $\mathbf{B}$. Scale bar in $\mathbf{C}=100 \mu \mathrm{m}$ and applies to (D). Scale bar in $\mathbf{E}=100 \mu \mathrm{m}$ and applies to (F,G). Amg, amygdala, Cd, caudate, $\mathrm{CL}$, claustrum, GP, globus pallidus, OT, optic tract, Put, putamen. approach toward novel, non-threatening, stimuli while the CNF initiates avoidance in response to stimuli that represent danger (Westby et al., 1990).

In contrast to what would be expected from their common name, most species of tree shrews (family Tupaiidae) are terrestrial and forage within $1.5 \mathrm{~m}$ of the ground ( Kawamichi and Kawamichi, 1979; Langham, 1982; Emmons, 2000). Predators vary depending on local region, but most always include mammalian carnivores and birds of prey. From her field studies, Emmons (2000) noted that tree shrews may appear indifferent to threats from below, but almost always emitted strong alarm signals to perceived threats from above. With these aspects of their behavior in mind, our previous findings of the projections of the visual field on the SC are relevant. Specifically, we found that the tree shrew SC projects topographically to the Pd and Pc, with the medial SC (upper visual field) projecting to the $\mathrm{Pd}$, and the lateral SC (lower visual field) projecting to the Pc ("specific" projections, schematically illustrated on the left side of Figure 6). The Pd receives additional non-topographic convergent input from the entire SC ("diffuse" projections, schematically illustrated on the right side of Figure 6). We suggested that specific tectopulvinar projections could function in coordinating precise movements to capture insects or fruit, while the diffuse tectopulvinar projections may mediate rapid responses to visual stimuli that would activate large regions of the visual field which would be considered threatening to tree shrews.

Our current results, which indicate that both the Pd and Pc project to the caudate and putamen (red arrows, left side of Figure 6) while the Pd, but not the Pc, projects to the amygdala (black arrow, right side of Figure 6) further support our proposed division of function of the tecto-pulvinar pathways through the $\mathrm{Pd}$ and Pc. Projections from the Pd and Pc to the GABAergic output neurons of the striatum could initiate inhibition of GABAergic nigrotectal cells which densely innervate PDB cells (Bickford and Hall, 1992). Disinhibition of PDB cells has been well established 


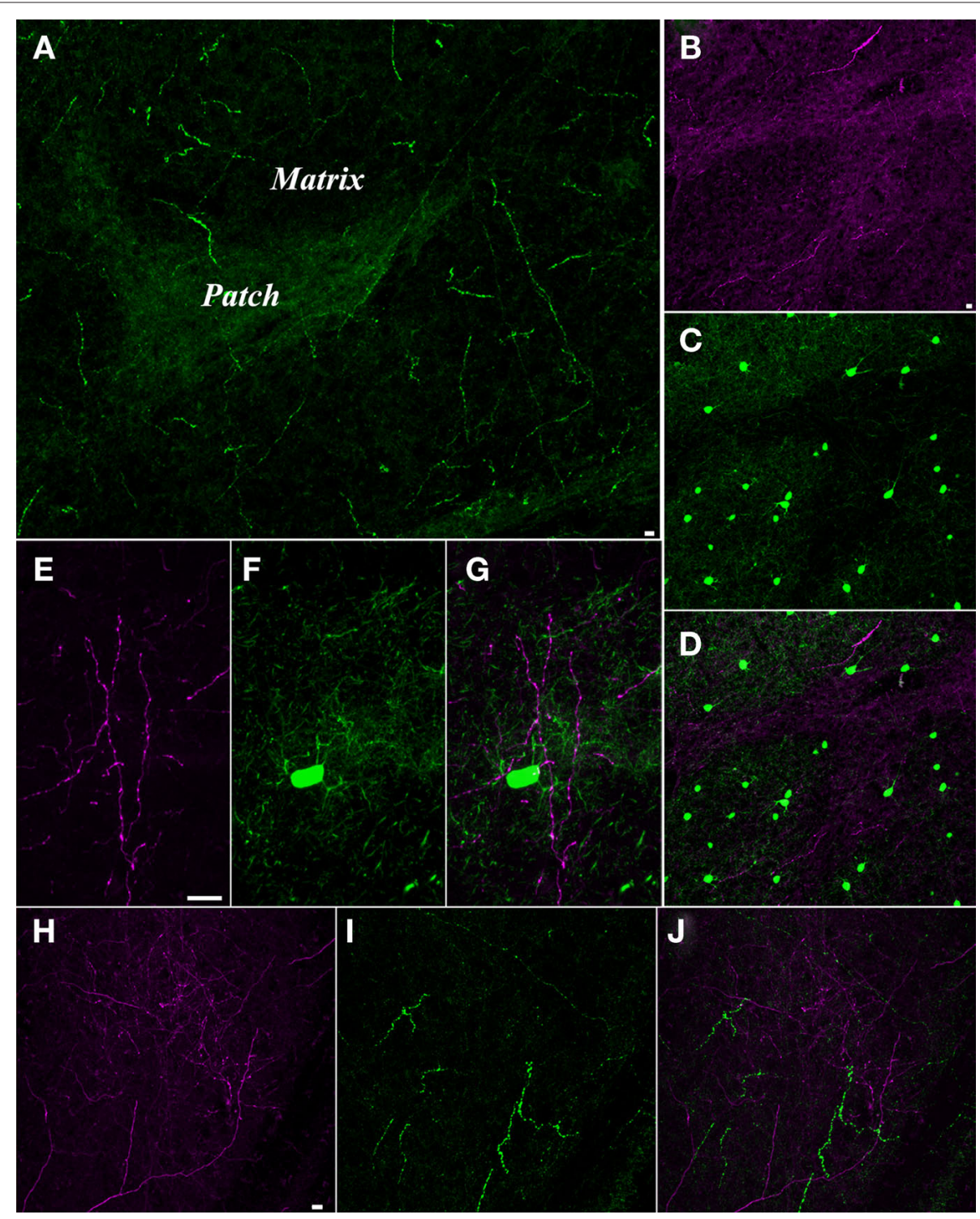

FIGURE 4 | Pulvinar axons innervate the striatum matrix. (A) Confocal microscope image of substance $P$ (SP, green) staining in the putamen. The matrix is sparsely innervated by thick SP axons, while the patches are densely innervated by fine SP axons. (B-D) Confocal image of a section stained for SP and parvalbumin (PV). The fine SP axons (purple, B) that innervate the patches interdigitate with a dense distribution of cells in the matrix that contain PV

(C). (D) Merged image of (B) and (C) illustrates the interlocking arrangement of the SP-rich patches and the PV-rich matrix. (E-G) Pulvino-striatal axons innervate the PV-rich matrix. E illustrates BDA-labeled pulvino-striatal axons (purple) in the putamen. (F) shows a PV stained cell and dendrites (green) in the same section. (G) Merged image of (E) and (F) shows that pulvino-striatal axons target the PV-rich matrix. (H-J) Pulvino-striatal axons innervate the SP-poor matrix. (H) shows BDA-labeled pulvino-strital axons (purple) in the putamen. (I) shows SP staining (green) in the same section. Only the sparse SP axons of the matrix are stained. (J) A merged image of (H) and (I) illustrates that pulvino-striatal axons innervate the SP-poor matrix. All scale bars $=10 \mu \mathrm{m}$. Scale bar in (B) also applies to (C,D). Scale bar in (E) also applies to (F,G). Scale bar in $\mathbf{( H )}$ also applies to $\mathbf{( I , J )}$. as a primary mechanism in the initiation of saccades and orienting movements, and the importance of SC-basal ganglia loops in this process has been clearly demonstrated (Hikosaka and Wurtz, 1983, 1985, 1989; Wurtz and Hikosaka, 1986; McHaffie et al., 2006; Schulz et al., 2009).

The Pd projections to the lateral amygdala (activated by diffuse tectopulvinar projections and/or specific projections from regions of the SC representing the upper visual field) could function to signal potential danger. A wide variety of studies have indicated that the amygdala is activated by threatening visual images (e.g., Morris et al., 2001; Vuilleumier et al., 2003; Liddell et al., 2005). As indicated on right side of Figure 6, the lateral amygdala ( $\mathrm{La}$ ) projects to both the striatum and the central amygdala (Ce, Amorapanth et al., 2000; LeDoux, 2007). The Ce projects densely to the dopaminergic substantia nigra pars compacta (Gonzales and Chesselet, 1990; Fudge and Haber, 2000) which may serve to amplify or inhibit subsequent motor responses, dependent on their context (Amorapanth et al., 2000; Dommett et al., 2005; Redgrave and Gurney, 2006; Cohen and Castro-Alamancos, 2010). 


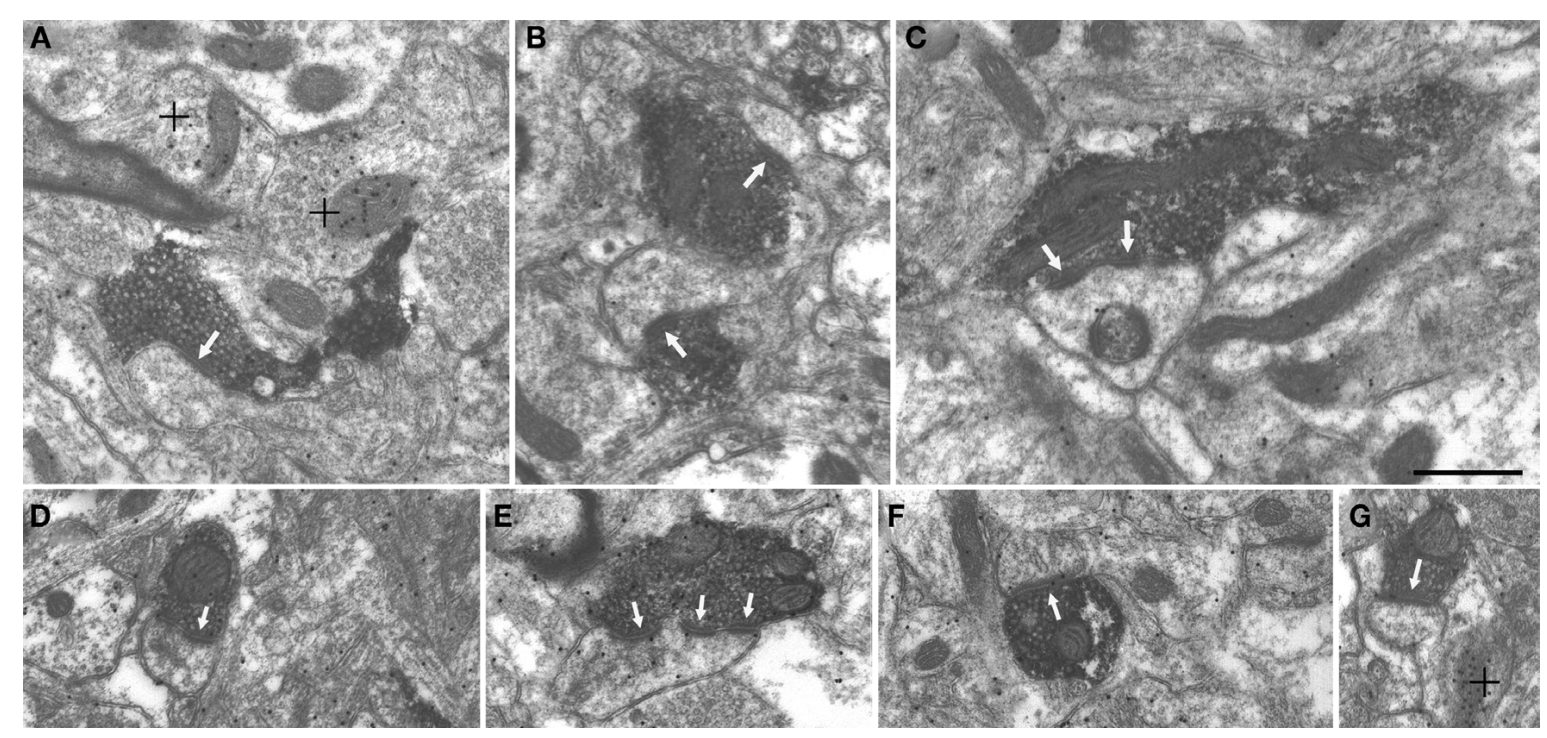

FIGURE 5 | Pulvino-striatal and pulvino-amygdala terminals contact spines. Terminals labeled by the anterograde transport of BDA injected in the pulvinar nucleus contact (white arrows) spines in the putamen $(\mathbf{A}-\mathbf{C}$ ) and the amygdala (D-G). Single (A,B,D,F,G) perforated (C) and multiple (E) contacts were observed. Postembedding immunocytochemical staining for GABA revealed surrounding GABAergic terminals (high density of gold particles; + denotes) but BDA-labeled terminals and postsynaptic spines contained a low density of gold particles. Scale bar $=0.5 \mu \mathrm{m}$ and applies to all panels.

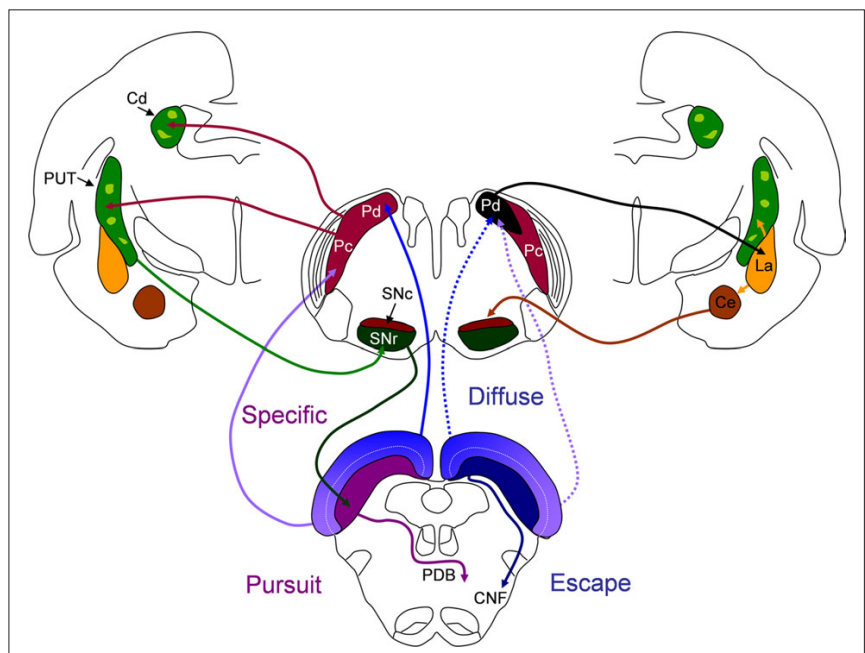

FIGURE 6 |The potential influence of dorsal (Pd) and central (Pc) pulvinar projections on basal ganglia circuits and behavior initiated by the superior colliculus (SC). Left side of figure: the $\mathrm{Pd}$ and $\mathrm{PC}$ receive topographically arranged inputs from the SC ("specific") and project to spiny output cells in the matrix (dark green) of the caudate $(\mathrm{Cd})$ and putamen (PUT). GABAergic spiny cells of the Cd and PUT project to the substantia nigra pars reticulate ( $\mathrm{SNr}$ ) and GABAergic nigrotectal cells densely innervate the cells of origin of the crossed predorsal bundle (PDB). Tecto-pulvinostriatal projections could initiate pursuit movements mediated by the PDB. Right side of figure: the $\mathrm{Pd}$ receives additional non-topographic, convergent projections from the SC ("diffuse") and projects to spiny output cells of the lateral amygdala (La). The La projects to the PUT and central amygdala (Ce). The Ce projects to the dopaminergic substantia nigra pars compacata (SNc). Tecto-pulvino-amygdala projections could serve to amplify escape movements mediated by the ipsilateral cuneiform (CNF) pathway. See text for details.

\section{VISUAL CAPABILITIES IN THE ABSENCE OF V1: RELATIONSHIP TO TECTO-PULVINAR PROJECTIONS}

The proportion of the visual thalamus innervated by the superficial (retinorecipient) layers of the SC varies with species. In nocturnal rodents, which rely primarily on somatosensory signals to navigate their environment, a relatively small region of the lateral posterior nucleus (LPN) receives input from the superficial SC and these projections are non-topographic (Mooney et al., 1984; Masterson et al., 2009). In contrast, in diurnal squirrels and tree shrews, fast moving species which rely heavily on visual information to navigate their habitats, the SC is proportionally larger and the superficial layers innervate relatively large regions of the pulvinar nucleus in a topographic manner (Robson and Hall, 1976, 1977; Luppino et al., 1988; Harting et al., 1991; Van Hooser and Nelson, 2006; Chomsung et al., 2008). These differences in tectothalamic innervation patterns are also reflected in the density of projections from the visual thalamus to the striatum. In rats, the tectorecipient LPN contributes relatively sparse input to the striatum (Doron and Ledoux, 1999; Cheatwood et al., 2003, 2005; Kamishina et al., 2008), while the tectorecipient pulvinar nucleus of the squirrel (Lin et al., 1984) and tree shrew projects densely to the striatum.

The density of tecto-pulvinar connections in the tree shrew and squirrel is likely related to their surprisingly robust visual capabilities in the absence of V1. Snyder et al. (1969) and Diamond and Hall (1969) tested tree shrew and squirrel visual behavior after complete ablation of the striate cortex (with corresponding degeneration of the lateral geniculate nucleus) and discovered that both species exhibited relatively normal visual behavior. Their detailed studies of the tree shrew, and others that followed (e.g., Snyder et al., 1969; Ward and Masterton, 1970; Killackey and Diamond, 1971; Killackey et al., 1971; Ware et al., 1972, 1974), 
revealed that tree shrews with V1 lesions were still able to make visual pattern discriminations, but that extension of the ablations to include the cortical targets of the pulvinar (i.e., temporal cortex) severely compromised the animals' visual ability and visual discrimination learning. Furthermore, ablations of the pulvinar targets alone (leaving striate areas intact) produced deficits in visual pattern discrimination and in visual "reversal learning" not seen following striate lesions. Because the temporal cortex lesions caused severe degeneration of the pulvinar nucleus, the pulvinostriatal and pulvino-amygdala projections were also likely affected in these experiments. Finally, lesions of the SC were found to compromise some color vision tasks in addition to producing deficits in pattern discrimination (Casagrande et al., 1972; Casagrande and Diamond, 1974). Taken together, these studies clearly demonstrate the significant role of the tecto-pulvinar system in many aspects of tree shrew vision.

In primates, $\mathrm{V} 1$ lesions produce a profound loss of visual perception, yet the phenomena of "blindsight" has revealed that even in humans, conscious perception of visual stimuli is not a prerequisite for the detection of visual stimuli and the initiation of reactive

\section{REFERENCES}

Amorapanth, P., LeDoux, J. E., and Nader, K. (2000). Different lateral amygdala outputs mediate reactions and actions elicited by a fear-arousing stimulus. Nat. Neurosci. 3, 74-79.

Arend, I., Machado, L., Ward, R., McGrath, M., Ro, T., and Rafal, R. D. (2008). The role of the human pulvinar in visual attention and action: evidence from temporal-order judgment, saccade decision, and antisaccade tasks. Prog. Brain Res. 171, 475-483.

Beckstead, R. M. (1984). The thalamostriatal projection in the cat. J. Comp. Neurol. 223, 313-346.

Berman, R. A., and Wurtz, R. H. (2008). Exploring the pulvinar path to visual cortex. Prog. Brain Res. 171, 467-473.

Berman, R. A., and Wurtz, R. H. (2010). Functional identification of a pulvinar path from superior colliculus to cortical area MT. J. Neurosci. 30, 6342-6354.

Bickford, M. E., and Hall, W. C. (1989). Collateral projections of predorsal bundle cells of the superior colliculus in the rat. J. Comp. Neurol. 283, 86-106.

Bickford, M.E., and Hall, W.C. (1992). The nigral projection to predorsal bundle cells in the superior colliculus of the rat. J. Comp. Neurol. 319, 11-33.

Casagrande, V. A., and Diamond, I. T. (1974).Ablation study of the superior colliculus in the tree shrew (Tupaia glis). J. Comp. Neurol. 156, 207-237.

Casagrande, V. A., Harting, J. K., Hall, W. C., Diamond, I. T., and Martin, G. F. (1972). Superior colliculus of the tree shrew: a structural and functional subdivision into superficial and deep layers. Science 177, 444-447.
Cheatwood, J.L., Corwin, J.V., and Reep, R. L. (2005). Overlap and interdigitation of cortical and thalamic afferents to dorsocentral striatum in the rat. Brain Res. 1036, 90-100.

Cheatwood, J. L., Reep, R. L., and Corwin, J. V. (2003). The associative striatum: cortical and thalamic projections to the dorsocentral striatum in rats. Brain Res. 968, 1-14.

Chomsung, R. D., Petry, H. M., and Bickford, M. E. (2008). Ultrastructural examination of diffuse and specific tectopulvinar projections in the tree shrew. J. Comp. Neurol. 510, 24-46.

Chomsung, R. D., Wei, H., Day-Brown, J. D., Petry, H. M., and Bickford, M. E. (2010). Synaptic organization of connections between the temporal cortex and pulvinar nucleus of the tree shrew. Cereb. Cortex 20, 997-1011.

Cohen, J. D., and Castro-Alamancos, M. A. (2010). Neural correlates of active avoidance behavior in superior colliculus. J. Neurosci. 30, 8502-8511.

Collins, C. E., Lyon, D. C., and Kaas, J. H. (2003). Responses of neurons in the middle temporal visual area after longstanding lesions of the primary visual cortex in adult new world monkeys. J. Neurosci. 23, 2251-2264.

Collins, C. E., Xu, X., Khaytin, I., Kaskan, P. M., Casagrande, V. A., and Kaas, J. H. (2005). Optical imaging of visually evoked responses in the middle temporal area after deactivation of primary visual cortex in adult primates. Proc. Natl. Acad. Sci. U.S.A. 102, 5594-5599.

Cowey, A. (2010). The blindsight saga. Exp. Brain Res. 200, 3-24. movements (Cowey, 2010). Although it is still unclear what pathways mediate blindsight, many studies suggest that projections from the pulvinar or dorsal lateral geniculate nucleus to the middle temporal cortex (area MT) are involved (Sincich et al., 2004; Berman and Wurtz, 2010; Lyon et al., 2010; Schmid et al., 2010). This idea remains controversial however, because V1 lesions severely diminish the responsiveness of area MT while the effects of SC lesions on area MT responses are relatively minor (Rodman et al., 1989, 1990; Girard et al., 1992; Kaas and Krubitzer, 1992; Collins et al., 2003, 2005). Furthermore, although inactivation of the pulvinar nucleus severely disrupts visually guided behavior (Wilke et al., 2010), it is still unclear whether this is primarily due to disruption of cortical or subcortical circuits. Our results raise the possibility that projections of the pulvinar nucleus to the striatum and amygdala contribute to the detection of visual signals and the initiation of appropriate behavioral responses, and may also play a role in blindsight.

\section{ACKNOWLEDGMENT}

Grant sponsor: NIH; Grant number EY016155; Grant number MH089867.

Dean, P., Redgrave, P., Sahibzada, N., and Tsuji, K. (1986). Head and body movements produced by electrical stimulation of superior colliculus in rats: effects of interruption of crossed tectoreticulospinal pathway. Neuroscience 19, 367-380.

Dean, P., Redgrave, P., and Westby, G. W. (1989). Event or emergency? Two response systems in the mammalian superior colliculus. Trends Neurosci. 12, 137-147.

Diamond, I. T., and Hall, W. C. (1969). Evolution of neocortex. Science 164, 251-262.

Dommett, E., Coizet, V., Blaha, C. D. Martindale, J., Lefebvre, V., Walton, N., Mayhew, J. E., Overton, P. G., and Redgrave, P. (2005). How visual stimuli activate dopaminergic neurons at short latency. Science 307, 1476-1479.

Doron, N. N., and Ledoux, J. E. (1999). Organization of projections to the lateral amygdala from auditory and visual areas of the thalamus in the rat. J. Comp. Neurol. 412, 383-409.

Emmons, L. H. (2000). Tupai: A Field Study of Bornean Treeshrews. Berkeley, CA: University of California Press.

Fudge, J. L., and Haber, S. N. (2000) The central nucleus of the amygdala projection to dopamine subpopulations in primates. Neuroscience 97 479-494.

Girard, P., Salin, P.A., and Bullier, J. (1992). Response selectivity of neurons in area MT of the macaque monkey during reversible inactivation of area V1. J. Neurophysiol. 67, 1437-1446.

Gonzales, C., and Chesselet, M. F. (1990). Amygdalonigral pathway: an anterograde study in the rat with Phaseolus vulgaris leucoagglutinin (PHA-L). J. Comp. Neurol. 297, 182-200.

Graybiel, A. M. (2008). Habits, rituals, and the evaluative brain. Annu. Rev. Neurosci. 31, 359-387.

Graybiel, A. M., Ragsdale, C. W. Jr. (1978). Histochemically distinct compartments in the striatum of human, monkeys, and cat demonstrated by acetylthiocholinesterase staining. Proc. Natl. Acad. Sci. U.S.A. 75, 5723-5726.

Grieve, K. L., Acuna, C., and Cudeiro, J. (2000). The primate pulvinar nuclei: vision and action. Trends Neurosci. 23, 35-39.

Harting, J. K., Huerta, M. F., Hashikawa, T., and van Lieshout, D. P. (1991) Projection of the mammalian superior colliculus upon the dorsal lateral geniculate nucleus: organization of tectogeniculate pathways in nineteen species. J. Comp. Neurol. 304, 275-306.

Harting, J. K., and Updyke, B. V. (2006). Oculomotor-related pathways of the basal ganglia. Prog. Brain Res. 151, 441-460.

Harting, J. K., Updyke, B. V., and Van Lieshout, D. P. (2001a). Striatal projections from the cat visual thalamus. Eur. J. Neurosci. 14, 893-896.

Harting, J. K., Updyke, B. V., and Van Lieshout, D. P. (2001b). The visualoculomotor striatum of the cat: functional relationship to the superior colliculus. Exp. Brain Res. 136, 138-142.

Hikosaka, O., and Wurtz, R. H. (1983). Visual and oculomotor functions of monkey substantia nigra pars reticulata. IV. Relation of substantia nigra 
to superior colliculus. J. Neurophysiol. 49, 1285-1301.

Hikosaka, O., and Wurtz, R. H. (1985). Modification of saccadic eye movements by GABA-related substances. II. Effects of muscimol in monkey substantia nigra pars reticulata. $J$. Neurophysiol. 53, 292-308.

Hikosaka, O., and Wurtz, R. H. (1989). The basal ganglia. Rev. Oculomot. Res. 3, 257-281.

Kaas, J. H., and Krubitzer, L. A. (1992). Area 17 lesions deactivate area MT in owl monkeys. Vis. Neurosci. 9, 399-407.

Kamishina, H., Yurcisin, G. H., Corwin, J. V., and Reep, R. L. (2008). Striatal projections from the rat lateral posterior thalamic nucleus. Brain Res. 1204, 24-39.

Karnath, H. O., Fruhmann Berger, M., Kuker, W., and Rorden, C. (2004). The anatomy of spatial neglect based on voxelwise statistical analysis: a study of 140 patients. Cereb. Cortex 14, 1164-1172.

Karnath, H. O., Himmelbach, M., and Rorden, C. (2002). The subcortical anatomy of human spatial neglect: putamen, caudate nucleus and pulvinar. Brain 125, 350-360.

Kawamichi, T., and Kawamichi, M. (1979). Spatial organization and territory of tree shrews (Tupaia glis). Anim. Behav. 27, 381-393

Kelly, L. R., Li, J., Carden, W. B., and Bickford, M.E. (2003). Ultrastructure and synaptic targets of tectothalamic terminals in the cat lateral posterior nucleus. J. Comp. Neurol. 464, 472-486.

Killackey, H., and Diamond, I. T. (1971). Visual attention in the tree shrew: an ablation study of the striate and extrastriate visual cortex. Science 171, 696-699.

Killackey, H., Snyder, M., and Diamond, I. T. (1971). Function of striate and temporal cortex in the tree shrew. J. Comp. Physiol. Psychol. 74(Suppl. 2), 1-29.

Kunzle, H. (2006). Thalamo-striatal projections in the hedgehog tenrec. Brain Res. 1100, 78-92.

Langham, N. P. E. (1982). The ecology of the common tree shrew, Tupaia glis, in peninsular Malaysia. J. Zool. Lond. 197, 323-344.

LeDoux, J. (2007). The amygdala. Curr. Biol. 17, R868-R874.

Lee, H. J., Groshek, F., Petrovich, G. D., Cantalini, J. P., Gallagher, M., and Holland, P. C. (2005). Role of amygdalo-nigral circuitry in conditioning of a visual stimulus paired with food. $J$. Neurosci. 25, 3881-3888.

Li, J., Wang, S., and Bickford, M.E. (2003). Comparison of the ultrastructure of cortical and retinal terminals in the rat dorsal lateral geniculate and lat- eral posterior nuclei. J. Comp. Neurol. 460, 394-409.

Liddell, B. J., Brown, K. J., Kemp, A. H., Barton, M. J., Das, P., Peduto, A., Gordon, E., and Williams, L. M. (2005). A direct brainstem-amygdala-cortical 'alarm' system for subliminal signals of fear. Neuroimage 24, 235-243.

Liddell, B. J., Williams, L. M., Rathjen, J., Shevrin, H., and Gordon, E. (2004). A temporal dissociation of subliminal versus supraliminal fear perception: an event-related potential study. J. Cogn. Neurosci. 16, 479-486.

Lin, C. S., May, P. J., and Hall, W.C. (1984). Nonintralaminar thalamostriatal projections in the gray squirrel (Sciurus carolinensis) and tree shrew (Tupaia glis). J. Comp. Neurol. 230, 33-46.

Linke, R., De Lima, A. D., Schwegler, H., and Pape, H.C. (1999). Direct synaptic connections of axons from superior colliculus with identified thalamoamygdaloid projection neurons in the rat: possible substrates of a subcortical visual pathway to the amygdala. $J$. Comp. Neurol. 403, 158-170.

Luppino, G., Matelli, M., Carey, R. G., Fitzpatrick, D., and Diamond, I. T. (1988). New view of the organization of the pulvinar nucleus in Tupaia as revealed by tectopulvinar and pulvinar-cortical projections. J. Comp. Neurol. 273, 67-86.

Lyon, D. C., Nassi, J.J., and Callaway, E. M. (2010). A disynaptic relay from superior colliculus to dorsal stream visual cortex in macaque monkey. Neuron $65,270-279$.

Masterson, S. P., Li, J., and Bickford, M. E. (2009). Synaptic organization of the tectorecipient zone of the rat lateral posterior nucleus. J. Comp. Neurol. $515,647-663$

McHaffie, J. G., Jiang, H., May, P. J., Coizet, V., Overton, P. G., Stein, B. E., and Redgrave, P. (2006). A direct projection from superior colliculus to substantia nigra pars compacta in the cat. Neuroscience 138, 221-234.

Mikula, S., Parrish, S. K., Trimmer, J. S., and Jones, E. G. (2009). Complete 3D visualization of primate striosomes by KChIP1 immunostaining. J. Comp. Neurol. 514, 507-517.

Mooney, R. D., Fish, S. E., and Rhoades, R. W. (1984). Anatomical and functional organization of pathway from superior colliculus to lateral posterior nucleus in hamster. J. Neurophysiol. 51, 407-431.

Morris, J. S., DeGelder, B., Weiskrantz, L., and Dolan, R. J. (2001). Differential extrageniculostriate and amygdala responses to presentation of emotional faces in a cortically blind field. Brain 124, 1241-1252.

Morris, J. S., Ohman, A., and Dolan, R. J. (1999). A subcortical pathway to the right amygdala mediating "unseen" fear. Proc. Natl. Acad. Sci. U.S.A. 96 , 1680-1685.

Patel, N. C., and Bickford, M. E. (1997). Synaptic targets of cholinergic terminals in the pulvinar nucleus of the cat. J. Comp. Neurol. 387, 266-278.

Redgrave, P., and Gurney, K. (2006). The short-latency dopamine signal: a role in discovering novel actions? Nat. Rev. Neurosci. 7, 967-975.

Redgrave, P., Odekunle, A., and Dean, P. (1986). Tectal cells of origin of predorsal bundle in rat: location and segregation from ipsilateral descending pathway. Exp. Brain Res. 63, 279-293.

Robson, J. A., and Hall, W. C. (1976). Projections from the superior colliculus to the dorsal lateral geniculate nucleus of the grey squirrel (Sciurus carolinensis). Brain Res. 113 , 379-385.

Robson, J. A., and Hall, W. C. (1977). The organization of the pulvinar in the grey squirrel (Sciurus carolinensis). I. Cytoarchitecture and connections. J. Comp. Neurol. 173, 355-388.

Rodman, H. R., Gross, C. G., and Albright, T. D. (1989). Afferent basis of visual response properties in area MT of the macaque. I. Effects of striate cortex removal. J. Neurosci. 9, 2033-2050.

Rodman, H. R., Gross, C. G., and Albright, T. D. (1990). Afferent basis of visual response properties in area MT of the macaque. II. Effects of superior colliculus removal. J. Neurosci. 10, 1154-1164.

Sahibzada, N., Dean, P., and Redgrave, P. (1986). Movements resembling orientation or avoidance elicited by electrical stimulation of the superior colliculus in rats. J. Neurosci. 6 , 723-733.

Schmid, M. C., Mrowka, S. W., Turchi, J., Saunders, R. C., Wilke, M., Peters, A. J., Ye, F. Q., and Leopold, D. A. (2010). Blindsight depends on the lateral geniculate nucleus. Nature. 466, 373-377.

Schulz, J. M., Redgrave, P., Mehring, C., Aertsen, A., Clements, K. M., Wickens, J. R., and Reynolds, J. N. (2009). Shortlatency activation of striatal spiny neurons via subcortical visual pathways. $J$. Neurosci. 29, 6336-6347.

Shi, C., and Davis, M. (2001). Visual pathways involved in fear conditioning measured with fear-potentiated startle: behavioral and anatomic studies. J. Neurosci. 21, 9844-9855.

Sincich, L. C., Park, K. F., Wohlgemuth, M. J., and Horton, J. C. (2004). Bypassing V1: a direct geniculate input to area MT. Nat. Neurosci. 7, 1123-1128.

Snow, J. C., Allen, H. A., Rafal, R. D., and Humphreys, G. W. (2009). Impaired attentional selection following lesions to human pulvinar: evidence for homology between human and monkey. Proc. Natl. Acad. Sci. U.S.A. 106, 4054-4059.

Snyder, M., Killackey, H., and Diamond, I. T. (1969). Color vision in the tree shrew after removal of posterior neocortex. J. Neurophysiol. 32, 554-563.

Stepniewska, I., and Kaas, J. H. (1997). Architectonic subdivisions of the inferior pulvinar in New World and Old World monkeys. Vis. Neurosci. 14 1043-1060.

Stepniewska, I., Qi, H. X., and Kaas, J. H. (1999). Do superior colliculus projection zones in the inferior pulvinar project to MT in primates? Eur. J. Neurosci. 11, 469-480.

Stepniewska, I., Qi, H. X., and Kaas, J. H. (2000). Projections of the superior colliculus to subdivisions of the inferior pulvinar in New World and Old World monkeys. Vis. Neurosci. 17, 529-549.

Takada, M., Itoh, K., Sugimoto, T., and Mizuno, N. (1985a). Topographical projections from the thalamus to the putamen in the cat. Neurosci. Lett. 54, 207-212.

Takada, M., Itoh, K., Yasui, Y., Sugimoto, T., and Mizuno, N. (1985b). Topographical projections from the posterior thalamic regions to the striatum in the cat, with reference to possible tecto-thalamo-striatal connections. Exp. Brain Res. 60, 385-396.

Van Hooser, S. D., and Nelson, S. B. (2006). The squirrel as a rodent model of the human visual system. Vis. Neurosci. 23, 765-778.

Verdon, V., Schwartz, S., Lovblad, K. O, Hauert, C. A., and Vuilleumier, P. (2010). Neuroanatomy of hemispatial neglect and its functional components: a study using voxel-based lesion-symptom mapping. Brain 133, 880-894.

Vuilleumier, P., Armony, J. L., Driver, J., and Dolan, R. J. (2003). Distinct spatial frequency sensitivities for processing faces and emotional expressions. Nat. Neurosci. 6, 624-631.

Ward, J. P., and Masterton, B. (1970). Encephalization and visual cortex in the Tree Shrew (Tupaia glis). Brain Behav. Evol. 3, 421-469.

Ward, R., Danziger, S., and Bamford, S. (2005). Response to visual threat following damage to the pulvinar. Curr. Biol. 15, 571-573.

Ware, C. B., Casagrande, V. A., and Diamond, I. T. (1972). Does the acuity of the tree shrew suffer from removal of striate cortex? A commentary on the paper by ward and Masterton. Brain Behav. Evol. 5, 18-29.

Ware, C. B., Diamond, I. T., and Casagrande, V. A. (1974). Effects of ablating the striate cortex on a successive pattern discrimination: further 
study of the visual system in the tree shrew (Tupaia glis). Brain Behav. Evol. 9, 264-279.

Westby, G. W., Keay, K. A., Redgrave, P., Dean, P., and Bannister, M. (1990). Output pathways from the rat superior colliculus mediating approach and avoidance have different sensory properties. Exp. Brain Res. 81, 626-638.

Wilke, M., Turchi, J., Smith, K., Mishkin, M., and Leopold, D. A. (2010). Pulvinar inactivation disrupts selection of movement plans. J. Neurosci. $30,8650-8659$.
Williams, L. M., Liddell, B. J., Kemp, A. H., Bryant, R. A., Meares, R. A., Peduto, A. S., and Gordon, E. (2006). Amygdalaprefrontal dissociation of subliminal and supraliminal fear. Hum. Brain Mapp. 27, 652-661.

Wong, P., Collins, C. E., Baldwin, M. K., and Kaas, J. H. (2009). Cortical connections of the visual pulvinar complex in prosimian galagos (Otolemur garnetti). J. Comp. Neurol. 517, 493-511.

Wurtz, R. H., and Hikosaka, O. (1986). Role of the basal ganglia in the initiation of saccadic eye movements. Prog. Brain Res. 64, 175-190.

Conflict of Interest Statement: The authors declare that the research was conducted in the absence of any commercial or financial relationships that could be construed as a potential conflict of interest.

Received: 29 June 2010; paper pending published: 10 August 2010; accepted: 04 October 2010; published online: 15 November 2010.
Citation: Day-Brown JD, Wei $H$, Chomsung RD, Petry HM and Bickford ME (2010) Pulvinar projections to the striatum and amygdala in the tree shrew. Front. Neuroanat. 4:143. doi: 10.3389/ fnana.2010.00143

Copyright (C) 2010 Day-brown, Wei, Chomsung, Petry and Bickford. This is an open-access article subject to an exclusive license agreement between the authors and the Frontiers Research Foundation, which permits unrestricted use, distribution, and reproduction in any medium, provided the original authors and source are credited. 